\title{
MicroRNA-577 inhibits gastric cancer growth by targeting E2F transcription factor 3
}

\author{
ZHANJIANG YU ${ }^{1}$, WEI ZHANG ${ }^{2}$ and FENGCHUN DENG ${ }^{3}$ \\ Departments of ${ }^{1}$ General Surgery and ${ }^{2}$ Endocrinology, The Third Affiliated Hospital of Qiqihar Medical University; \\ ${ }^{3}$ Department of Anatomy, Qiqihar Medical University, Qiqihar, Heilongjiang 161006, P.R. China
}

Received June 23, 2014; Accepted March 24, 2015

DOI: $10.3892 / \mathrm{ol} .2015 .3390$

\begin{abstract}
The incidence and mortality rates of gastric cancer are one of the highest of all types of cancers. Emerging evidence has demonstrated that altered expression of micro (mi)RNAs may be implicated in the tumorigenesis of numerous types of cancer. Therefore, miRNAs may have potential as important tools in cancer diagnostics and therapeutics. miRNAs regulate the expression of genes involved in mediating cell proliferation and developmental timing, among numerous other processes. Altered expression levels of miRNAs may result in the ability of cells to proliferate aberrantly and migrate. The present study used reverse transcription-quantitative polymerase chain reaction assays to analyze miRNA-577 expression in gastric cancer tissues and cell lines, MTT and cell cycle analysis to examine cell proliferation in vitro, and luciferase assays and western blot to investigate miRNA-577's downstream targets. The results demonstrated that miRNA-577 was significantly downregulated in gastric cancer patient samples and cell lines. In addition, miRNA-577 affected an important regulator of E2F transcription factor 3 expression and that altered miRNA-577 expression resulted in the aberrant proliferation of gastric cancer cells.
\end{abstract}

\section{Introduction}

Gastric cancer (GC) ranks the fifth most prevalent type of cancer and the third leading cause of cancer-associated mortality worldwide, accounting for 952,000 novel diagnoses (6.8\% incidence) and 723,000 mortalities in 2012 (1). GC in developing countries accounts for $>70 \%$ of worldwide total cases; currently China, Japan and Korea account for $60 \%$ of total cases $(2,3)$. The USA has the lowest incidence of GC,

Correspondence to: Dr Fengchun Deng, Department of Anatomy, Qiqihar Medical University, 333 Bukui Street, Qiqihar, Heilongjiang 161006, P.R. China

E-mail: fengchundeng@163.com

Key words: microRNA-577, gastric cancer, E2F transcription factor 3, cell growth comprising $1.3 \%(\sim 22,000)$ and $1.9 \%(\sim 11,000)$ of all novel cancer cases and cancer-associated mortalities, respectively, in 2014 (4).

Small non-coding RNAs, known as microRNAs (miRNA), regulate gene expression at the post-transcriptional level by binding to complementary messenger (m)RNA sequences, primarily in the 3'-untranslated region (UTR) of the target. Subsequently, target mRNA is degraded or its translation into protein is inhibited $(5,6)$. miRNAs have been implicated in numerous biological processes, including cell proliferation, metabolism, apoptosis, morphogenesis, developmental timing and stress-response (7-9). In relation to cancer, miRNAs have been reported to be differentially expressed, acting as oncogenes as well as tumor suppressors $(10,11)$; in addition, numerous miRNA may have the potential for use as diagnostic and prognostic markers in cancer as well as possible therapeutic targets (12-17).

A limited number of previous studies have investigated the role of miRNA (miR)-577 in cancer. One such study reported that miR-577 regulated testis-specific gene 10 protein expression, inactivated the $\mathrm{p} 53$ and retinoblastoma protein pathways as well as modulated the $G_{1} / S$ phase transition, leading to an increased rate of malignancy and progression of esophageal squamous cell carcinomas (18). In GC patients, miR-577 expression was reported to correlate with chemosensitivity (19), possibly through modulating cytochrome P450 protein expression (20). E2F3 is a member of the E2F family of transcription factors and is important in the control of cell cycle during tumor development including GC (21). Several miRNA have been demonstrated to repress tumor growth by regulating E2F3 (22).

The present study aimed to investigate the expression of miR-577 in GC tissues and cell lines in order to provide evidence for miR-577 as a diagnostic and prognostic marker of $\mathrm{GC}$ as well as a potential therapeutic target.

\section{Materials and methods}

Samples. The present study was approved by the Medical Ethics Committee of Qiqihar Medical University (Qiqihar, China). None of the patients in the study received chemotherapy or radiation treatment prior to surgery. Written informed consent was obtained from all patients. GC and adjacent normal tissues ( $\geq 5 \mathrm{~cm}$ from the edge of the tumor) were collected by 
debulking surgery from 30 patients who were recruited into a clinical trial at the Third Affiliated Hospital of Qiqihar Medical University between 2012 and 2013. Clinicopathologic information was obtained and two pathologists independently determined diagnoses according to the International Union Against Cancer (7th edition).

Cell lines. Human GC cell lines HGC-27, SGC-7901, MKN-28, NCI-N87 and MKN-74 as well as the immortalized human gastric epithelial mucosa cell line GES1 were purchased from the Cell Bank of Type Culture Collection of Chinese Academy of Sciences (Shanghai, China). All cell lines were cultured in Dulbecco's modified Eagle's medium supplemented with $10 \%$ fetal bovine serum, $100 \mathrm{U} / \mathrm{ml}$ penicillin and $100 \mu \mathrm{g} / \mathrm{ml}$ streptomycin (all purchased from Gibco Life Technologies, Grand Island, NY, USA). All cells were cultured at $37^{\circ} \mathrm{C}$ in a $5 \% \mathrm{CO}_{2}$ atmosphere.

RNA extraction and reverse transcription quantitative polymerase chain reaction ( $R T-q P C R)$. Total miRNA from cultured cells and surgical GC tissues was extracted using TRIzol ${ }^{\circledR}$ reagent (Invitrogen Life Technologies, Carlsbad, CA, USA) according to the manufacturer's instructions. Complementary DNA was synthesized from 5 ng total RNA using the TaqMan miRNA reverse transcription kit (Invitrogen Life Technologies). Expression levels of miR-577 were quantified by qPCR using the reverse transcription products, TaqMan 2 Universal PCR Master Mix without UNG Amperase (Applied Biosystems Life Technologies, Foster City, CA, USA), miRNA-specific TaqMan probes, and primers (Applied Biosystems) on a 7500 Fast Real Time PCR system (Applied Biosystems) with an initial denaturation at $95^{\circ} \mathrm{C}$, followed by 40 cycles at $95^{\circ} \mathrm{C}$ for $15 \mathrm{~s}$ and $60^{\circ} \mathrm{C}$ for $1 \mathrm{~min}$. The threshold cycle (CT) was then determined and defined as the fractional cycle number at which the fluorescence detected passes a fixed threshold. The Applied Biosystems 7500 Fast software (Applied Biosystems) was used to analyze the CT values of different miRNAs normalized to an endogenous control U6. The normalized values (dCT) from tumorous tissue were then compared with its paired nontumorous tissue, yielding miRNA differential expression profiles. Primers were used as follows: miR-577 F 5'-ACA CTCCAGCTGGGTAGATAAAATATTGG-3' and R 5'-CTC AACTGGTGTCGTGGAGTCGGCAATTCAGTTGAGCAG GTACC-3'; U6, 5'-CTCGCTTCGGCAGCACA-3', R 5-AAC GCTTCACGAATTTGCGT-3'. miRNA mimics, small interfering (si)RNA and plasmids. miR-577, anti-miR-577 and control miRNA encoding plasmids were purchased from Shanghai GenePharma Co., Ltd (Shanghai, China). E2F transcription factor 3 (E2F3)-small interfering (si)RNA and scrambled control siRNA were purchased from Santa Cruz Biotechnology, Inc. (Dallas, TX, USA). siRNA and miRNA transfection were performed using Lipofectamine 2000 (Invitrogen Life Technologies) according to the manufacturer's instructions. The Renilla and firefly luciferase activities in cell lysates were measured $48 \mathrm{~h}$ after transfection using the dual-luciferase reporter assay system (Promega Corporation, Madison, WI, USA), and the results were presented as the ratio of Renilla activity/firefly activity in the lysate. Renilla activity was used to normalize the relative luciferase values. Each transfection was performed in triplicate.
Cell proliferation assay. Cell viability was measured using an MTS assay kit (Promega Corp.), as described previously (23). Briefly, at 2 days post transfection, 2,000 cells/well were seeded into 96-well plates and following $24 \mathrm{~h}$, the medium was replaced with fresh medium and the cells were further cultured for 1, 2 or 3 days. Following culture, MTS $(20 \mu \mathrm{l})$ solution was added to each well and plates were incubated for $1 \mathrm{~h}$ at $37^{\circ} \mathrm{C}$. Absorbance (A) was recorded at $490 \mathrm{~nm}$ using a 3500 microplate reader (Bio-Rad Laboratories, Inc., Richmond, CA, USA). Each individual experiment was performed in six replicates for three independent times.

Cell cycle analysis. Cells $\left(2 \times 10^{6} / \mathrm{ml}\right)$ were transfected with miR-577, control miRNA or E2F3 siRNA and cultured for 2 days. Cells were then collected, washed twice with phosphate-buffered saline and fixed with ice-cold $70 \%$ ethanol, then incubated with RNase I (1 $\mu \mathrm{g} / \mathrm{ml}$; Sigma-Aldrich, St. Louis, MO, USA) at $37^{\circ} \mathrm{C}$ for $1 \mathrm{~h}$. Samples were analyzed using a flow cytometer (LSRII; BD Biosciences, San Jose, CA, USA) following the addition of propidium iodide $(20 \mu \mathrm{g} / \mathrm{ml})$.

Protein isolation and western blot analysis. Total proteins were extracted using radioimmunopreciptiation assay lysis buffer with proteinase/phosphatase inhibitors (Thermo Fisher Scientific, Cambridge, MA, USA). Lysate was separated using $10 \%$ sodium dodecyl sulfate polyacrylamide gel electrophoresis (Bio-Rad Laboratories, Inc., Hercules, CA, USA), which were then blotted onto polyvinylidene difluoride membranes (Millipore, Bedford, MA, USA). The membrane was blocked in 5\% non fat milk (Fisher Scientific Co., Fair Lawn, NJ, USA) for $1 \mathrm{~h}$ and subsequently incubated with either rabbit polyclonal antibody against human E2F3 (sc-878; 1:500 dilution) or goat polyclonal antibody against human GAPDH (sc-20357; 1:1,000 dilution) at $4^{\circ} \mathrm{C}$ overnight. The membrane was washed 3 times and then treated with horseradish peroxidase-conjugated mouse anti-rabbit or anti-goat second antibodies (1:10,000; Santa Cruz Biotechnology, Inc.) for $1 \mathrm{~h}$ at room temperature. The bands were detected using an enhanced chemiluminescence kit (Thermo Fisher Scientific).

Statistical analysis. Values are expressed as the mean \pm standard error of the mean. Differences between groups were analyzed using a one way analysis of variance followed by Bonferroni post-hoc analyses, as appropriate. All data were analyzed using SPSS software, version 19.0 (IBM SPSS, Armonk, NY, USA). $\mathrm{P}<0.05$ was considered to indicate a statistically significant difference between values.

\section{Results}

miR-577 is downregulated in human GC tissues and cell lines. In order to investigate the role of miR-577 in human GC, miR-577 expression was analyzed in GC patient samples and adjacent healthy tissue controls. The results demonstrated that miR-577 was significantly downregulated in 24 out of $30 \mathrm{GC}$ patient samples compared with paired control samples $(\mathrm{P}<0.01)$ (Fig. 1A). To further evaluate the association of miR-577 and GC, miR-577 expression was examined in GC cancer cell lines using RT-qPCR. The results revealed that miR-577 expression was significantly decreased in all 
A

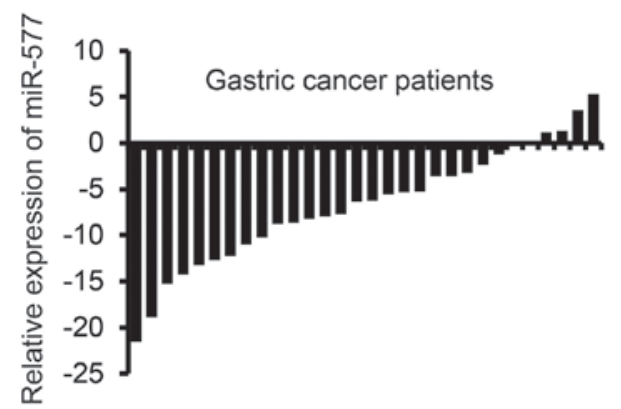

B

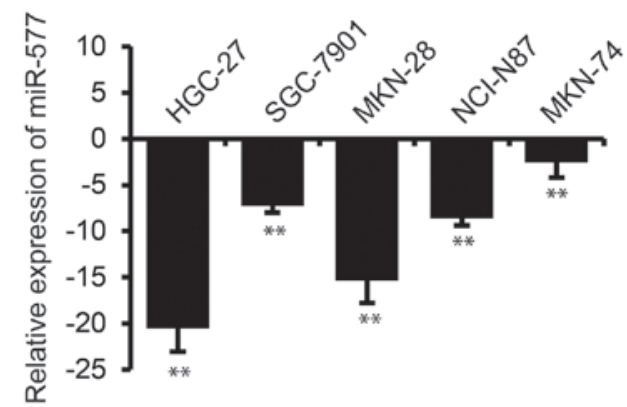

Figure 1. Downregulation of miR-577 expression in human GC patient tissues and cell lines. (A) Reverse transcription quantitative polymerase chain reaction analysis of miR-577 expression in primary GC tissues. Transcript levels were normalized to U6 expression and data are presented as the relative expression compared with paired adjacent normal tissue $(n=30)$. (B) Expression levels of miR-577 were examined in five GC cell lines. Transcript levels were normalized to U6 expression and data are presented the relative expression compared with normal GES1 gastric mucosa cell. Values are presented as the mean \pm standard error of the mean of three independent experiments. ${ }^{* *} \mathrm{P}<0.01$ vs. GES1 cells. miR, microRNA; GC, gastric cancer.

A

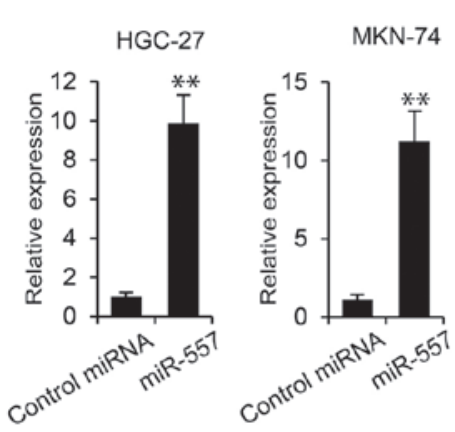

C

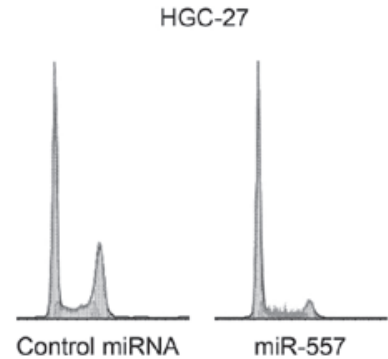

B

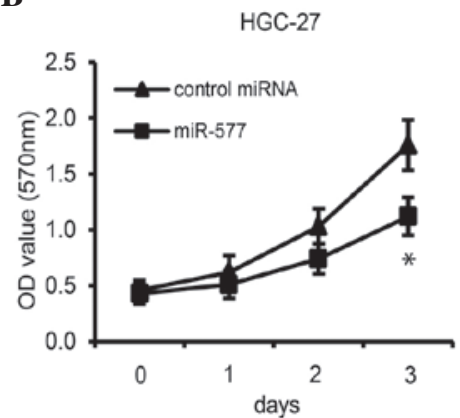

MKN-74

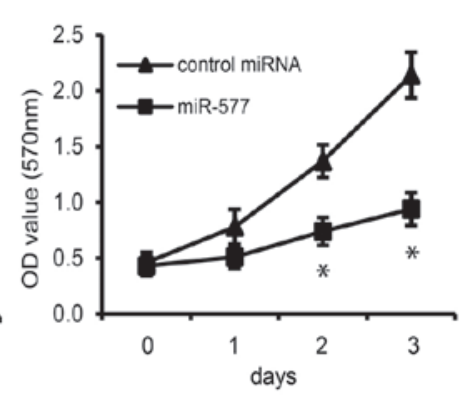

MKN-74
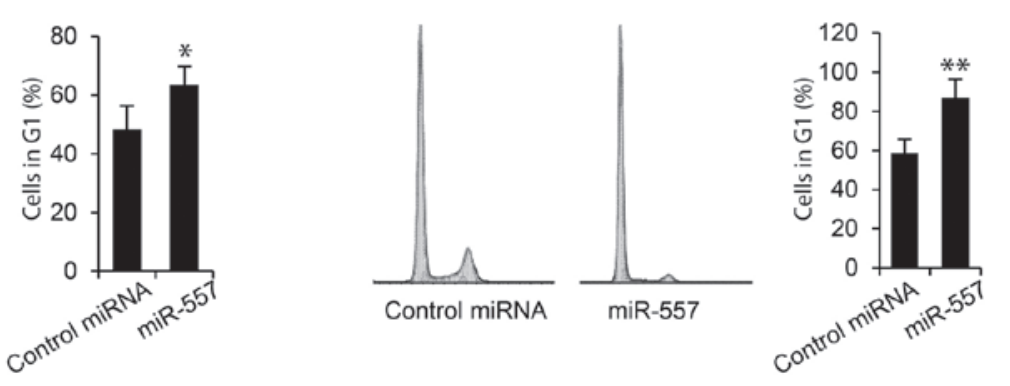

Figure 2. miR-577 overexpression inhibits gastric cancer cell growth. (A) Reverse transcription quantitative polymerase chain reaction analysis of relative miR-577 expression in control miRNA- and miR-577-transfected HGC-27 and MKN-74 cells at 2 days post transfection. Transcript levels were normalized to U6 expression and data are presented as the relative expression compared with control miRNA-transfected cells. (B) HGC-27 and MKN-74 cell proliferation was determined using MTS assays. (C) Cell cycle profiles of control miRNA- and miR-577-transfected HGC-27 and MKN-74 cells at two days post transfection. Values are presented as the mean \pm standard error of the mean of three independent experiments ( $\mathrm{n}=3$ ). $\mathrm{P}<0.05 \mathrm{vs}$. control miRNA. miR/miRNA, microRNA; OD, optical density.

five cell lines (HGC-27, SGC-7901, MKN-28, NCI-N87 and MKN-74) derived from GC, compared with the normal GES1 gastric mucosa cell line (Fig. 1B). These data therefore suggested that miR-577 expression was downregulated in GC.

miR-577 inhibits GC cell proliferation. In order to determine the role of miR-577 in GC tumorigenesis, the effect of miR-577 overexpression on HGC-27 and MKN-74 GC cell proliferation was examined. Cells were transfected with miR-577 or control miRNA. RT-qPCR revealed that miR-577 expression levels were significantly increased in cells transfected with miR-577 in each GC cell line (Fig. 2A). In addition, an MTS assay demonstrated that the overexpression of miR-577 inhibited proliferation of HGC-27 and MKN-74 cells (Fig. 2B). Furthermore, flow cytometric analysis was performed on transfected cells in order to determine cell cycle progression. As shown in Fig. 2C, transfection with miR-577 significantly increased the percentage of HGC-27 and MKN-74 cells in $\mathrm{G}_{1}$ phase $(\mathrm{P}<0.05$ and $\mathrm{P}<0.01)$; in addition, $\mathrm{S}$ phase peaks were decreased in miR-577-transfected cells. These results indicated that miR-577 inhibited GC cell growth.

miR-577 directly targets E2F3. In order to elucidate the biological implications of miR-577 on GC tumorigenesis, 


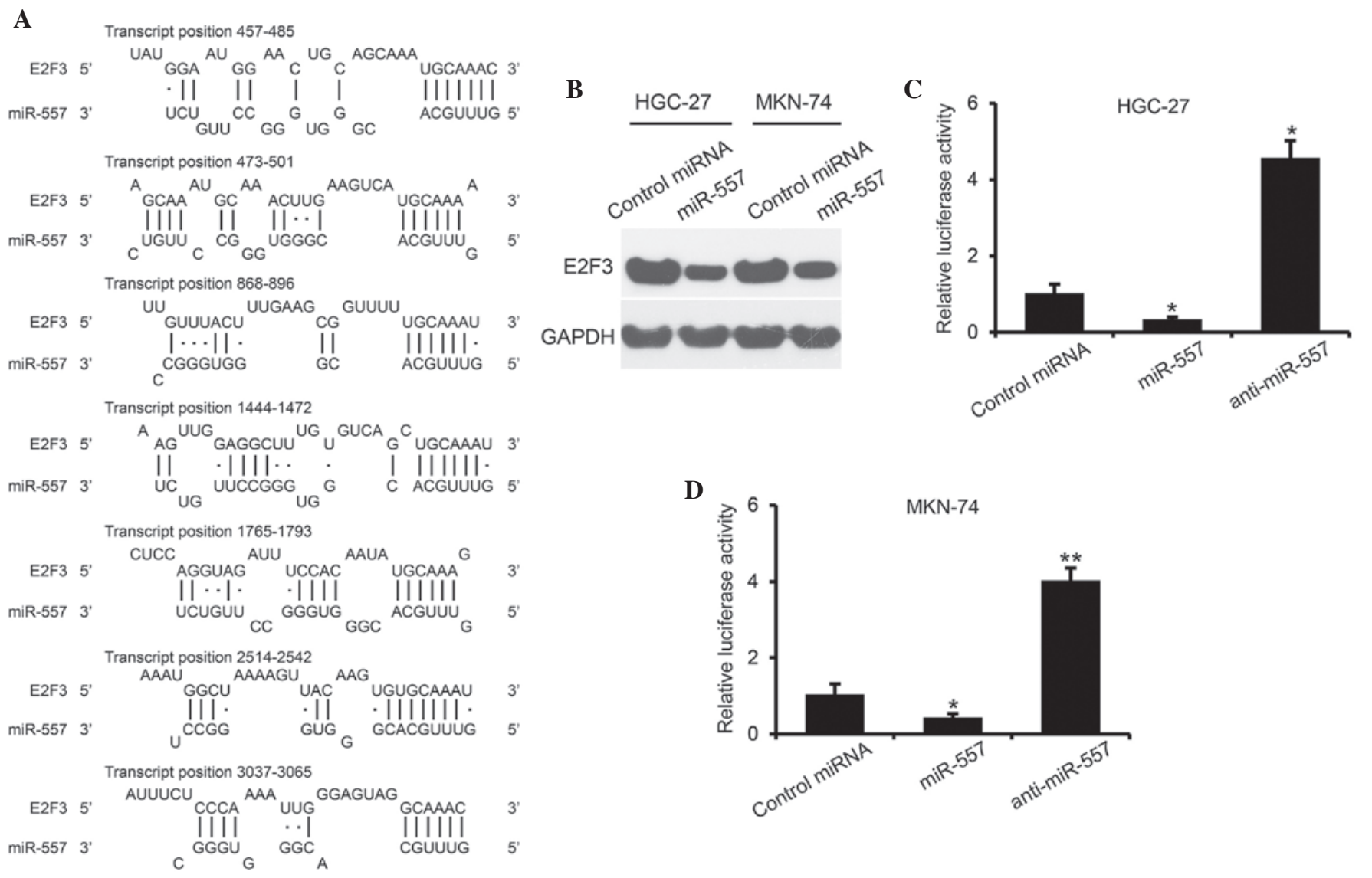

Figure 3. E2F3 is a direct target of miR-577. (A) Schematic representation of seven miR-577 putative target sites in E2F3 3'-UTR. (B) Western blot analysis of E2F3 protein levels in response to miR-577 overexpression in HGC-27 (left) and MKN-74 (right) cells. GAPDH was used as loading control. Relative luciferase activity of (C) HGC-27 and (D) MKN-74 cells transfected with plasmids carrying 3'-UTR of E2F3 gene and control miRNA, miR-577 or anti-miR577. Values are presented as the mean \pm standard error of the mean of three independent experiments $(n=4)$. ${ }^{*}<0.05$ and ${ }^{* *} \mathrm{P}<0.01$ vs. control miRNA. E2F3, E2F transcription factor 3; miR/miRNA, microRNA; UTR, untranslated region.

A

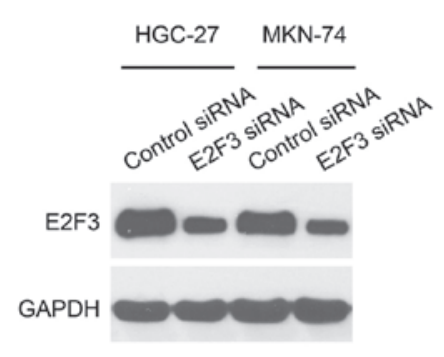

C

HGC-27

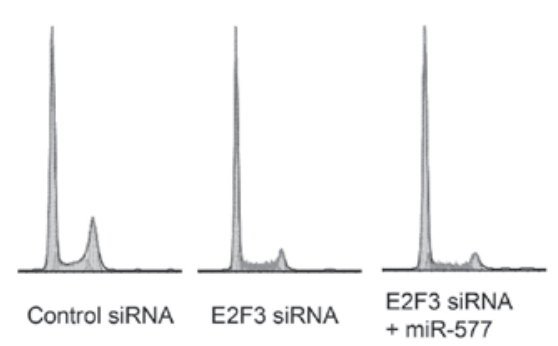

B

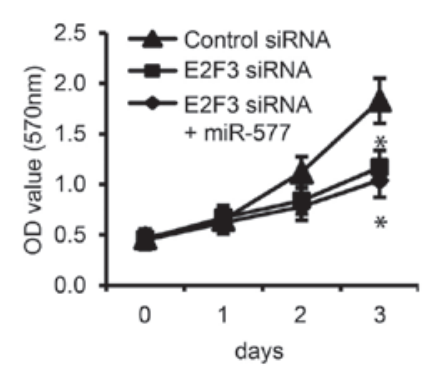

MKN-74

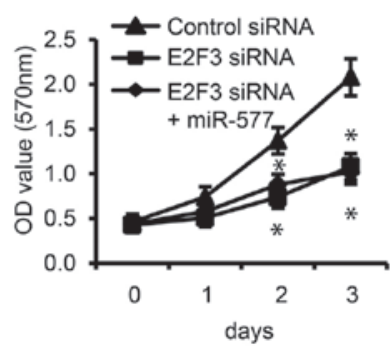

MKN-74
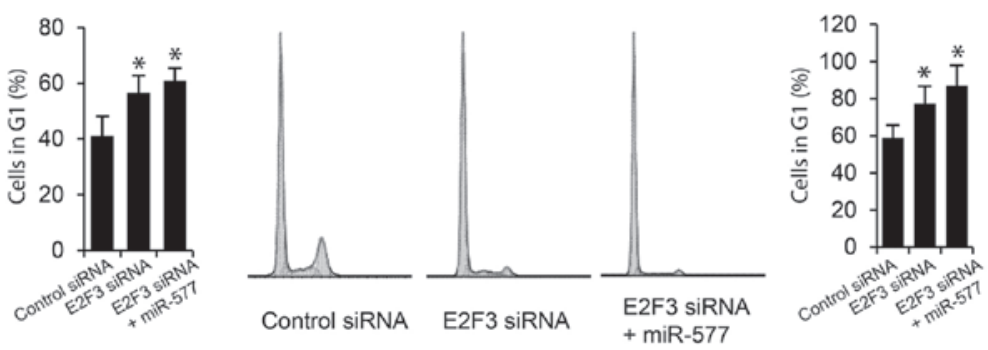

Figure 4. miR-577 inhibits gastric cancer cell proliferation by downregulating E2F3. (A) Western blot analysis of E2F3 protein levels in response to E2F3 siRNA in HGC-27 (left) and MKN-74 (right) cells. (B) Cell proliferation was determined using MTS assays in HGC-27 and MKN-74 cells transfected with control siRNA, E2F3 siRNA and E2F3 siRNA+miR-577. (C) Cell cycle profiles of control siRNA-, E2F3 siRNA- and E2F3 siRNA+miR-577-transfected HGC-27 and MKN-74 cells. Values are presented as the mean \pm standard error of the mean of three independent experiments $(n=4)$. "P<0.05 vs. control siRNA-transfected cells. E2F3, E2F transcription factor 3; miR, microRNA; siRNA, small interfering RNA; OD, optical density. 
TargetScan human 6.2 (www.targetscan.org), DNA Intelligent Analysis (diana.cslab.ece.ntua.gr) and MiRanda software -aug2010 (www.microrna.org) were used for putative human protein-coding gene targets of miR-577. The results revealed seven putative binding sites of miR-577, which are broadly conserved in vertebrates E2F3 3'-UTR (Fig. 3A). In order to confirm that miR-577 targets the E2F3 3'-UTR, HGC-27 and MKN-74 cells were transiently transfected with miR-577 and control miRNA. Western blot analysis revealed that expression of E2F3 was substantially decreased at $48 \mathrm{~h}$ following miR-577 transfection in each GC cell line (Fig. 3B). Furthermore, a luciferase reporter assay was performed, in which wild-type full length 3'-UTR of E2F3 was cloned downstream of the luciferase gene in a pGL3 vector. The results demonstrated that miR-577, rather than control miRNA, significantly suppressed the luciferase activity of reporter genes containing E2F3 3'-UTR in HGC-27 and MKN-74 cells ( $\mathrm{P}<0.05)$ (Fig. 3C and D). Furthermore, luciferase activity was increased $\sim 4$-fold compared with control miRNA in HGC-27 and MKN-74 cells transfected with anti-miR-577 ( $\mathrm{P}<0.05$ and 0.01 , respectively). These results suggested that $E 2 F 3$ is a direct target of miR-577.

miR-577 inhibits cell growth by suppressing E2F3 expression. In order to further investigate the role of E2F3 repression in miR-577-inhibited GC proliferation, the effects of E2F3 downregulation on GC cell proliferation were investigated. As shown in Fig. 4A, E2F3 silencing by specific E2F3 siRNAs significantly decreased E2F3 protein levels in HGC-27 and MKN-74 cells. In addition, downregulation of E2F3 markedly suppressed HGC-27 and MKN-74 cell proliferation. However, further overexpression of miR-577 in the E2F3-silenced cells exhibited no additive effect on cell proliferation, as determined using MTS assays and cell cycle analysis (Fig. 4B and C). These results demonstrated that E2F3 expression correlated with GC cell proliferation and indicated that E2F3 may be required for GC cells to proliferate; in addition, the expression of E2F3 was suggested to be regulated by miR-577.

\section{Discussion}

In the present study, miR-577 expression was analyzed in human GC tissue samples and cell lines, the results of which demonstrated a significant reduction in miR-577 expression in the majority of tissue samples and in all tested cell lines compared with adjacent health tissue and GES1 control cells, respectively. In addition, the role of miR-577 in cell proliferation was investigated; overexpression of miR-577 significantly inhibited proliferation as well as increased the percentage of cells in $\mathrm{G}_{1}$ phase and decrease the percentage of cells in $\mathrm{S}$ phase. During the $G_{1}$ phase, cells grow and express mRNA and proteins involved in mitosis; $\mathrm{S}$ phase follows, which involves DNA replication in preparation for cell division. The transition from $\mathrm{G}_{1}$ to $\mathrm{S}$ phase is a major checkpoint in cell cycle regulation, which is commonly disrupted in cancers $(24,25)$. The results of the present study demonstrated that miR-577 expression significantly affected the $G_{1}$ to $S$ phase transition in GC cells.

Based on the computational prediction of likely targets for miR-577, an expression study and luciferase reporter assay were conducted in order to investigate the regulation of E2F3 transcription by miR-577. The results revealed that miR-577 directly targeted and inhibited E2F3 expression. In addition, silencing E2F3 with siRNA demonstrated that downregulation of this transcription factor significantly suppressed the proliferation of GC cells.

The transcription factor E2F3 modulates expression of proteins involved in cell/centrosome cycles and mitosis and is critical for cellular proliferation (25). In addition, the E2F-family of transcription factors were reported to control gene expression vital to angiogenesis, extracellular matrix remodeling, tumor cell survival and interactions with vascular endothelial cells, which facilitates metastasis to the lungs (26). Expression of several genes that control the timing of $G_{1}$ to $\mathrm{S}$ phase transition, the rate of DNA synthesis and act as rate limiting factors of cellular proliferation, have been reported to be dependent on the presence of E2F3 (27-30). Increased expression of this transcription factor has been reported in other types of cancer and has been associated with altered miRNA expression $(26,29,31,32)$. These previous studies are consistent with the findings of the present study, which indicated that the downregulation of miR-577 allows for increased E2F3 expression, leading to aberrant cell proliferation.

In conclusion, the results of the present study demonstrated that downregulated expression of miR-577 in GC affected core mechanisms in the regulation of cell cycle progression, resulting in aberrant proliferative ability. In addition, the differential expression of miR-577 in GC allowed for the increased expression of the transcription factor E2F3, which was proposed to result in the increased transcription of factors required for $G_{1}$ to $S$ phase transition. These results enhance the current understanding of oncogenesis in GC and may potentially be used to develop novel diagnostic and therapeutic applications for GC treatment.

\section{Acknowledgements}

The present study was supported by the Department of Education Science and Technology Research Project in Heilongjiang Province (12531830).

\section{References}

1. Globocan 2012: Estimated Cancer Incidence, Mortality and Prevalence Worldwide in 2012. http://globocan.iarc. fr/Pages/fact_sheets_cancer.aspx. Accessed March 31, 2014.

2. Fock KM: Review article: The epidemiology and prevention of gastric cancer. Aliment Pharmacol Ther 40: 250-260, 2014.

3. Ferlay J, Soerjomataram I, Ervik M, et al; International Agency for Research on Cancer: GLOBOCAN 2012: Cancer incidence, mortality and prevalence worldwide in 2012. http://globocan.iarc.fr. Accessed November 20, 2014.

4. Howlader N, Noone AM, Krapcho M, et al; National Cancer Institute: SEER Cancer statistics review, 1975-2011. http://seer.cancer.gov/csr/1975_2011/. Accessed June 21, 2014.

5. Makeyev EV and Maniatis T: Multilevel regulation of gene expression by microRNAs. Science 319: 1789-1790, 2008.

6. Bushati N and Cohen SM: microRNA functions. Annu Rev Cell Dev Biol 23: 175-205, 2007.

7. Alvarez-Garcia I and Miska EA: MicroRNA functions in animal development and human disease. Development 132: 4653-4662, 2005

8. Leung AK and Sharp PA: MicroRNA functions in stress responses. Mol Cell 40: 205-215, 2010.

9. Yan B, Zhao LH, Guo JT and Zhao JL: miR-429 regulation of osmotic stress transcription factor 1 (OSTF1) in tilapia during osmotic stress. Biochem Biophys Res Commun 426: 294-298, 2012. 
10. DeSano JT and Xu L: MicroRNA regulation of cancer stem cells and therapeutic implications. AAPS J 11: 682-692, 2009.

11. Esquela-Kerscher A and Slack FJ: Oncomirs-microRNAs with a role in cancer. Nat Rev Cancer 6: 259-269, 2006.

12. Zhu W, He J, Chen D, et al: Expression of miR-29c, miR-93 and miR-429 as potential biomarkers for detection of early stage non-small lung cancer. PLoS One 9: e87780, 2014.

13. Schultz NA, Dehlendorff C, Jensen BV, et al: MicroRNA biomarkers in whole blood for detection of pancreatic cancer. JAMA 311: 392-404, 2014.

14. Ahmed FE, Ahmed NC, Vos PW, et al: Diagnostic microRNA markers to screen for sporadic human colon cancer in stool: I. Proof of principle. Cancer Genomics Proteomics 10 93-113, 2013

15. Li J, Du L, Yang Y, et al: MiR-429 is an independent prognostic factor in colorectal cancer and exerts its anti-apoptotic function by targeting SOX2. Cancer Lett 329: 84-90, 2013.

16. Wang Y, Li M, Zang W, et al: MiR-429 up-regulation induces apoptosis and suppresses invasion by targeting Bcl-2 and SP-1 in esophageal carcinoma. Cell Oncol (Dordr) 36: 385-394, 2013.

17. Krützfeldt J, Rajewsky N, Braich R, et al: Silencing of microRNAs in vivo with 'antagomirs'. Nature 438: 685-689, 2005.

18. Yuan X, He J, Sun F and Gu J: Effects and interactions of MiR-577 and TSGA10 in regulating esophageal squamous cell carcinoma. Int J Clin Exp Pathol 6: 2651-2667, 2013.

19. Kim CH, Kim HK, Rettig RL, et al: miRNA signature associated with outcome of gastric cancer patients following chemotherapy. BMC Med Genomics 4: 79, 2011.

20. Wei Z, Jiang S, Zhang Y, et al: The effect of microRNAs in the regulation of human CYP3A4: A systematic study using a mathematical model. Sci Rep 4: 4283, 2014.

21. Suzuki T, Yasui W, Yokozaki H, Naka K, Ishikawa T and Tahara E: Expression of the E2F family in human gastrointestinal carcinomas. Int J Cancer 81: 535-538, 1999.

22. Ciafre SA and Galardi S: microRNAs and RNA-binding proteins: A complex network of interactions and reciprocal regulations in cancer. RNA biol 10: 935-942, 2013.
23. Lai JP, Chien JR, Moser DR, et al: hSulf1 Sulfatase promotes apoptosis of hepatocellular cancer cells by decreasing heparin-binding growth factor signaling. Gastroenterology 126:23 $1-248,2004$

24. Nojima H: G1 and S-phase checkpoints, chromosome instability, and cancer. Methods Mol Biol 280: 3-49, 2004.

25. Humbert PO, Verona R, Trimarchi JM, Rogers C, Dandapani S and Lees JA: E2f3 is critical for normal cellular proliferation. Genes Dev 14: 690-703, 2000. Bartek J and Lukas J: Mammalian G1- and S-phase checkpoints in response to DNA damage. Curr Opin Cell Biol 13: 738-747, 2001.

26. Lee MY, Moreno CS and Saavedra HI: E2F activators signal and maintain centrosome amplification in breast cancer cells. Mol Cell Biol 34: 2581-2599, 2014.

27. Nevins JR: The Rb/E2F pathway and cancer. Hum Mol Genet 10: 699-703, 2001

28. Xiao F, Zhang W, Chen L, et al: MicroRNA-503 inhibits the G1/S transition by downregulating cyclin D3 and E2F3 in hepatocellular carcinoma. J Transl Med 11: 195, 2013.

29. Leone G, DeGregori J, Yan Z, et al: E2F3 activity is regulated during the cell cycle and is required for the induction of $\mathrm{S}$ phase. Genes Dev 12: 2120-2130, 1998.

30. Olsson AY, Feber A, Edwards S, et al: Role of E2F3 expression in modulating cellular proliferation rate in human bladder and prostate cancer cells. Oncogene 26: 1028-1037, 2007.

31. Ren XS, Yin MH, Zhang X, et al: Tumor-suppressive microRNA-449a induces growth arrest and senescence by targeting E2F3 in human lung cancer cells. Cancer Lett 344: 195-203, 2014.

32. Hollern DP, Honeysett J, Cardiff RD and Andrechek ER: The E2F transcription factors regulate tumor development and metastasis in a mouse model of metastatic breast cancer. Mol Cell Biol 34: 3229-3243, 2014. 